\title{
AN ANALYTICAL MODEL FOR WATER PROFILE CALCULATIONS IN FREE SURFACE FLOWS THROUGH ROCKFILLS
}

\author{
Amel Soualmia, Manel Jouini \\ Laboratory of Water Sciences and Technology, National Institute of Agronomy of Tunisia, University of Carthage, Tunisia \\ e-mail: amel.inat@hotmail.fr \\ Lucien Masbernat, Denis Dartus \\ Institute of fluid Mechanics of Toulouse, National Polytechnic Institute of Toulouse, Toulose, France
}

\begin{abstract}
In this study, an analytical model to resolve the water profiles equation of free surface flows through riprap has been developed. This model is based on Stephenson's relation to determine the head loss for different flow states in order to see its ability to reproduce the water profiles. This analytical model has been applied to experimental series realized in a rectangular channel built at the National Agronomic Institute of Tunisia (INAT) in collaboration with engineering consultants of the firm MECATER and in partnership with the INP Toulouse. MECATER is interested in these flows within the framework of a project with the nickel company SLN of ERAMET group in New Caledonia, concerning the protection of mining sterols against water flows at high mountains.
\end{abstract}

Keywords: analytical model, experimental channel, rockfill, simulations, Stephenson's formula

\section{Introduction}

This study concerns the problem of mine tailings storage out of water. This storage is protected from flooding by ensuring water flow through rockfill drains, called "wicks". This work has been conducted at the Sciences and Technology Water Laboratory (LSTE) of the National Agronomic Institute of Tunis (INA Tunis) in collaboration with the international firm of engineering consultants MECATER under contract with the Nickel Company of ERAMET group in New Caledonia, and in partnership with the Fluids Mechanics Institute of Toulouse IMF Toulouse, (Jouini, 2012; Soualmia et al., 2013).

This study refers to flows in wicks and considers Stephenson's formula (Prasad, 1970; Stephenson, 1979) to determine water depth, which is based on geometrical and physical parameters of rocky media. The aim of this work is to verify the ability of Stephenson's formula to reproduce the water profile in large porous media. The flow regime in a riprap is generally turbulent, which limits Darcy's relation (Bordier and Zimmer, 2000). It is then necessary to analyze the hydrodynamics in an experimental channel.

In order to achieve these objectives, firstly, a literature review allowed the analysis of the most commonly used formulas to characterize the non-linear relation between the hydraulic gradient and the flow infiltration velocity through the riprap (Michioku et al., 2005). For each formula, the parameters characterizing the riprap should be specified, such as the average hydraulic radius, porosity and particle size (Pagliara and Lotti, 2009).

Secondly, different runs have been made in the channel designed and built at the Sciences and Technology Water Laboratory (LSTE) of the National Agronomic Institute of Tunisia (INAT) within the frame of this study. The variation of water profiles in the riprap has been described 
using these tests (Soualmia et al., 2013) under the influence of various parameters such as flow discharge, porosity, particles diameter and channel bed slope (Bari and Hansen, 2002).

Then an analytical model developed in this work, based on Stephenson's formula, has been validated and applied to these experimental runs to illustrate the prediction of water profiles.

\section{Experimental set-up and measuring equipment}

\subsection{Experimental set-up}

Laboratory experiments have been carried out in the rectangular channel built at INAT, the length, height and width of which are $10 \mathrm{~m} \times 0.8 \mathrm{~m} \times 0.6 \mathrm{~m}$. The side walls are made of glass to enable observation of the flow. The top of the channel is made of sheet with few glass windows through which stones are filled and emptied. The structure is balanced on sheet metal supports. The slope of the channel varies between 0 and $15 \%$, and the adjustment thereof is effected by changing the height of the supports. The upstream channel is connected to a reservoir with capacity of $3.6 \mathrm{~m}^{3}$ through flexible rubber, which allows reaching high slopes. Inside this upstream tank, a honeycomb grid is installed horizontally to reduce the turbulence and water level fluctuations through the channel. The channel discharges downstream to a tank with a capacity of $9 \mathrm{~m}^{3}$, and the downstream channel can be closed (fully or partially) by a slide valve which would provide a closed conduit flow. The channel is fed by a closed circuit consisting of three centrifugal pumps supplying the elevated reservoir. The supply pipe to the channel is equipped with an electromagnetic flow meter for measuring the flow rate. Water is pumped from the downstream tank by this group of three pumps, each of which produces flow of $10 \mathrm{l} / \mathrm{s}$ to the upstream reservoir that feeds the channel. To prevent mobility of the riprap, the inlet and outlet are closed by two wire grids.

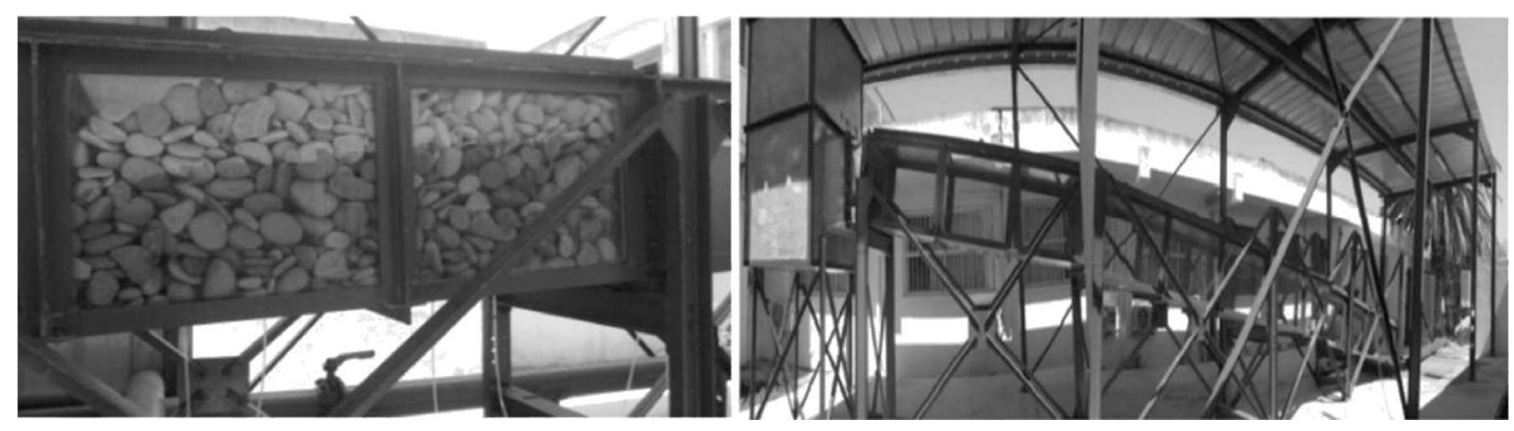

Fig. 1. The experimental channel built at INAT

\subsection{Measuring equipment}

The flow measurement is realized by an electromagnetic flow meter connected in the conduct. To measure water levels through the gravel during testing, seventeen piezometers are incorporated along the channel lower face. The spacing between the piezometers is $0.5 \mathrm{~m}$.

The speed measurement near the inlet and the outlet of the wick is carried out by microvane current meter.

\section{Experimental protocol}

The experiments consist on studying flows through stones placed in the central portion of the channel for different flow rates. These tests are renewed for different sizes of stones and different slopes. In each test, the flow velocity is measured at the inlet and the outlet of the wick, and also the evolution of the water profile is registerd. 
There are two types of stones, sharp stones for which there are three different sizes, and smooth stones.

We also determined the porosity of different kinds of stones in the laboratory.

Various tests presented in this study are defined in Table 1. In Fig. 2 different kinds of investigated stones are presented.
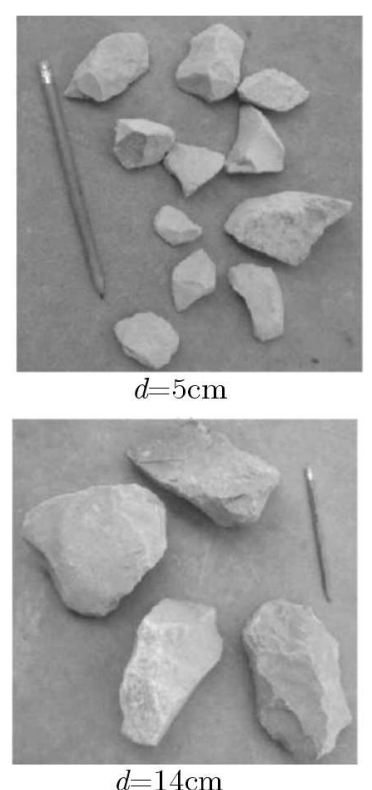

Fig. 2. Different types and sizes of investigated stones
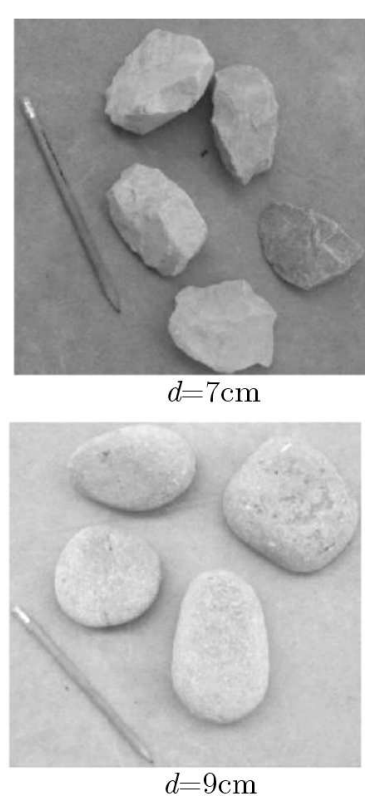

\section{Materials and methods}

\subsection{The free surface flow equations}

By assuming the gradually varied open channel flow the water profile equation will be given in the following form (Bari and Hansen, 2002)

$$
\frac{d h}{d x}=\frac{I-J}{1-F_{r p}^{2}}
$$

In Eq. (4.1), for a rectangular channel with width $B$ and water depth $h$, the pore Froude number Fr is expressed as

$$
\operatorname{Fr}_{p}=\frac{q}{n g \sqrt{h^{3}}}
$$

where $q=Q / B$ is the flow per unit of width $\left[\mathrm{m}^{2} \mathrm{~s}^{-2}\right]$ and $n$ is porosity of the wick.

In the carried out approach, the hydraulic gradient is expressed by Stephenson's formula (Stephenson, 1979)

$$
J=\frac{K_{s t} V^{2}}{g d n^{2}}
$$

where the parameter $K_{s t}$ is calculated by the following expression

$$
K_{s t}=\frac{\alpha_{0}}{\operatorname{Re}_{p}}+K_{t}
$$




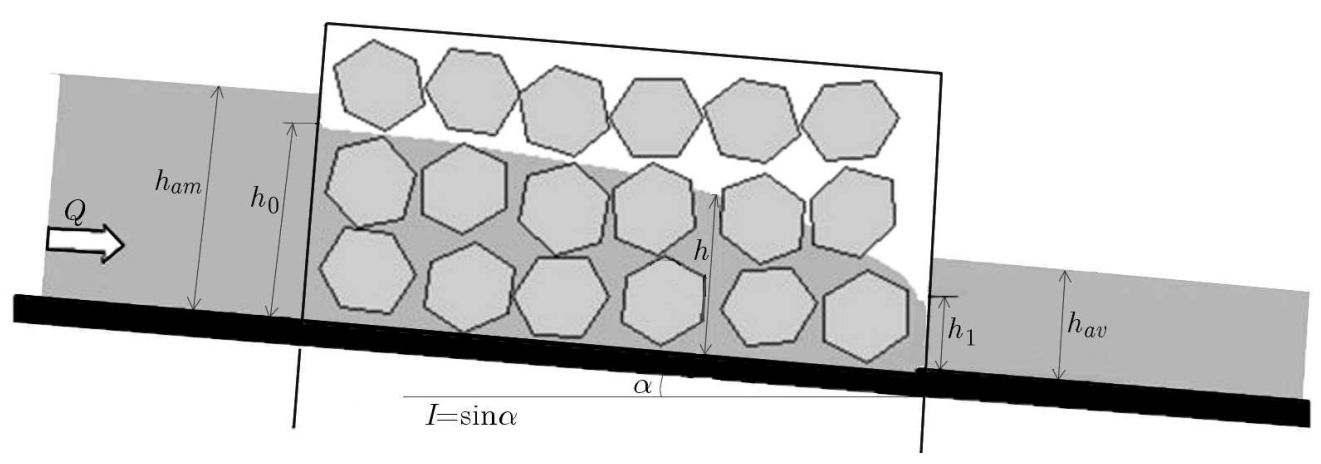

Fig. 3. Diagram of free surface flow through rockfill in a channel

The Reynolds number of the interstitial mean flow is defined by

$$
\operatorname{Re}_{p}=\frac{V_{p} d}{\nu}=\frac{V d}{n \nu}
$$

where $V_{p}=V / n$ is the water velocity through the voids.

The characteristic length $d$ is the average size of stones which constitute the wick. The constant $\alpha_{0}$ is usually equal to 800 .

We introduce dimensionless parameters using the critical height $h_{c}$ as the reference. This is given by

$$
h_{c}=\sqrt[3]{\frac{q^{2}}{n^{2} g}}
$$

The draft $h$, the longitudinal abscissa $x$ and the length $\mathrm{d}$ are expressed in dimensionless form by

$$
h^{*}=\frac{h}{h_{c}} \quad x^{*}=\frac{x}{h_{c}} \quad d^{*}=\frac{d}{h_{c}}
$$

The equation of the water profile is written as

$$
\frac{d h^{*}}{d x^{*}}=\frac{I h^{*}}{h^{* 3}-1}\left(h^{* 2}-\gamma h^{*}-m\right)
$$

Both parameters $m$ and $\gamma$ are given by the expressions

$$
m=\frac{K_{t}}{I d^{*}} \quad \gamma=\frac{\alpha_{0} \nu n}{I d^{* 2} q}
$$

They reflect the effect of the head loss: the parameter $m$ reflects the energy dissipation at high values of Reynolds number for which the flow could be turbulent in the wick. The parameter $\gamma$ corresponds to the linear head loss. It is low when the Reynolds number is high.

\section{The analytical model}

\subsection{Resolution of flow equation}

The calculations have been made with the upstream initial condition.

$$
x^{*}=0 \quad h^{*}=h_{0}^{*}
$$


Equation (4.8) is solved as $x^{*}=x^{*}\left(h^{*}\right)$ by writing

$$
I d x^{*}=\frac{\left(h^{* 3}-1\right) d h^{*}}{h^{*}\left(h^{* 2}-\gamma h^{*}-m\right)}
$$

To integrate (5.2), we first write it in the following form

$$
I d x^{*}=\frac{\left(h^{* 3}-1\right) d h}{h^{*}\left(h^{*}-h_{a}^{*}\right)\left(h^{*}-h_{b}^{*}\right)}=\left(1+\frac{A}{h^{*}}+\frac{B}{h^{*}-h_{a}^{*}}+\frac{C}{h^{*}-h_{b}^{*}}\right) d h^{*}
$$

where

$$
h_{a}^{*}=\frac{1}{2}\left(\gamma+\sqrt{\gamma^{2}+4 m}\right) \quad h_{b}^{*}=\frac{1}{2}\left(\gamma-\sqrt{\gamma^{2}+4 m}\right)
$$

and $A, B, C$, are given by

$$
\begin{aligned}
& A=\frac{1}{m} \quad B=\frac{1}{h_{a}^{*}-h_{b}^{*}}\left[h_{a}^{*}\left(\gamma-\frac{1}{m}\right)+\left(m+\frac{\gamma}{m}\right)\right] \\
& C=\frac{1}{h_{b}^{*}-h_{a}^{*}}\left[h_{b}^{*}\left(\gamma-\frac{1}{m}\right)+\left(m+\frac{\gamma}{m}\right)\right]
\end{aligned}
$$

The integration of (5.3), considering upstream conditions (5.1), leads to the following solution

$$
I x^{*}=h^{*}-h_{0}^{*}+A L_{m} \frac{h^{*}}{h_{0}^{*}}+B L_{m}\left|\frac{h^{*}-h_{a}^{*}}{h_{0}^{*}-h_{a}^{*}}\right|+C L_{m}\left|\frac{h^{*}-h_{b}^{*}}{h_{0}^{*}-h_{b}^{*}}\right|
$$

In the graphical representation, the $x$-coordinate is normalized by the length of the wick $L_{m}$, and calculated by $(5.6)$

$$
\xi=\frac{x}{L_{m}}=\frac{h_{C}}{I L_{m}} I x^{*}
$$

\section{Simulation results of the realized experiments}

\begin{tabular}{|c|c|c|c|c|c|c|c|c|c|}
\hline Test & $\begin{array}{c}d \\
{[\mathrm{~m}]}\end{array}$ & $n$ & $I$ & $\begin{array}{l}L_{m} \\
{[\mathrm{~m}]}\end{array}$ & $\begin{array}{c}\text { Test } \\
\text { number }\end{array}$ & $\begin{array}{c}Q \\
{[1 / \mathrm{s}]}\end{array}$ & $\begin{array}{c}h_{0} \\
{[\mathrm{~m}]}\end{array}$ & $\begin{array}{c}h_{c} \\
{[\mathrm{~m}]}\end{array}$ & $K t$ \\
\hline \multirow{3}{*}{ I } & \multirow{3}{*}{0.07} & \multirow{3}{*}{0.496} & \multirow{3}{*}{0.05} & \multirow{3}{*}{5} & $\overline{~ I-1}$ & 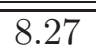 & 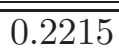 & $\overline{0.035}$ & 4.0000 \\
\hline & & & & & I-2 & 18.04 & 0.442 & 0.059 & 4.0000 \\
\hline & & & & & I-3 & 22.6 & 0.58 & 0.069 & 5.5000 \\
\hline \multirow{3}{*}{ II } & \multirow{3}{*}{0.09} & \multirow{3}{*}{0.426} & \multirow{3}{*}{0.05} & \multirow{3}{*}{2} & II-1 & 8.151 & 0.175 & 0.038 & 2.8500 \\
\hline & & & & & II-2 & 17.8 & 0.320 & 0.065 & 2.7200 \\
\hline & & & & & II-3 & 24.58 & 0.412 & 0.080 & 2.7800 \\
\hline
\end{tabular}

The previous solution has been applied to the first experimental series led at INAT, where the channel bed slope is $5 \%$.

We give below simulation results of the first and second tests which are defined in Table 1. These tests are a part of our first experiment series led in the rectangular channel.

Table 1. Experimental results (definition of the tests) and corresponding simulations (angularity parameter $K t$ )

The height of the wick input $h_{0}$ have been measured in each test. 

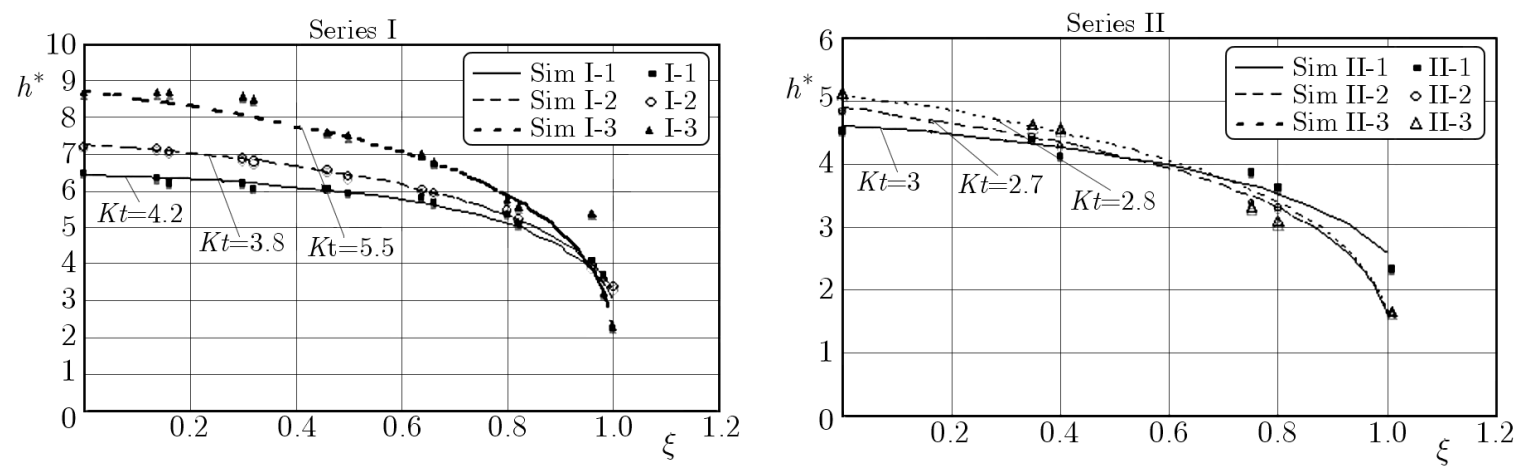

Fig. 4. Water profile calculations for test series I (pebbles diameter $d=7 \mathrm{~cm}$ ) and test series II $(d=9 \mathrm{~cm})$

We have to note that we solve the problem with the upstream initial condition $h_{0}$ because for the last downstream part of the wick we noticed that the gradually varied flow theorem is not valid anymore due to the presence of flow acceleration observed in this part.

The results are illustrated in Fig. 4.

We observed that in all experiments, it was possible to achieve satisfactory smoothing of water profiles by adjusting values of the angularity parameter $K t$.

We also noticed sensitivity of the results to the particle diameter. For example, in the case of the largest discharge, variation of $28.6 \%$ in the diameter leads to variation of about $70 \%$ in the water level.

In Fig. 5, the sensitivity of the tests to the parameter $K t$ is shown (for tests I-3).

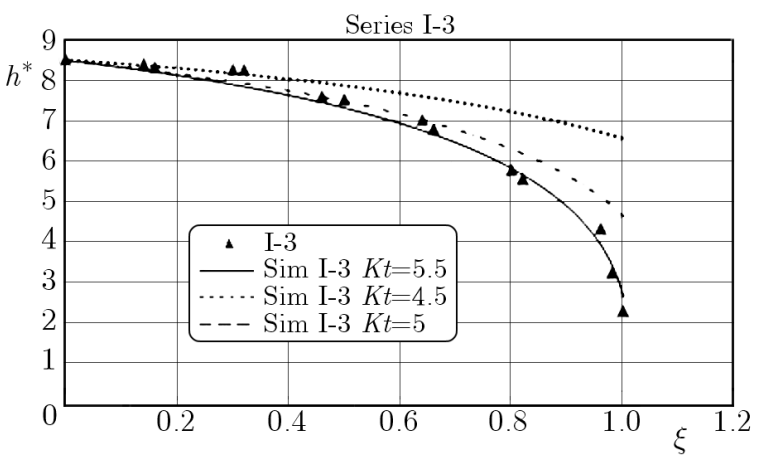

Fig. 5. Sensitivity of tests of the water profile to $K t$ values (tests I-3)

From the above results (Fig. 5) we can deduce that variation of $K t$ affects the water profiles at the downstream wick. In fact, the Stephenson formula is sensitive to this adjustment parameter that depends on stones angularity.

Finally, we want to underline that this analytical model allows acceptable prediction of water profiles by adjusting the parameter values $K t$.

\section{Conclusions}

The purpose of development of this analytical model is to verify the ability of the ahead loss expression, such as Stephenson's one, to reproduce the water profile for different flow states. We can consider that this aim is reached. In fact we obtained satisfactory results of water profile simulations, in comparison to experimental tests by adjusting the parameter $K t$. 
We also noted sensitivity of the results to variation of the experimental values of stone areas constituting the wick, especially to the size d of the stones. This suggests that efforts should be made in determining the characteristic properties of the studied media with more precision.

Finally, this analytical model can be considered useful to study such complex flows.

Acknowledgments

We would like to thank the Nickel Company (SLN) of ERAMET group in New Caledonia and MECATER's responsibility, especially Mr. Samir Ennour, Mr. Okba Boughanmi. We also thank Professor Elies Hamza (director of INAT) for his encouragement and attention he has paid on the implementation of the experimental channel.

\section{References}

1. BARi R., Hansen D., 2002, Application of gradually-varied flow algorithms to simulate buried streams, Journal of Hydraulic Research, 40, 6, 673-683

2. Bordier C., Zimmer D., 2000, Drainage equations and non-darcian modeling in coarse porous media or geosynthetic materials, Journal of Hydrology, 228, 3, 174-187

3. Chow V.T., 1959, Open Channel Hydraulics, McGraw-Hill Book Co, New York, 217-296

4. Jouini M., 2012, Study of flow in a wick stones in channel, Master thesis, National Agronomic Institute of Tunis (INAT), Tunisia

5. Jouini M., Soualmia A., SaAd K., 2013, Etude des écoulements dans une mèche en pierre, 11th Mechanical Congress, Agadir, Maroc, 103-105

6. Michioku K., Maeno S., Furusawa T., Haneda M., 2005, Discharge through a permeable rubble mound weir, Journal of Hydraulic Engineering, 131, 1, 1-10

7. Pagliara S., Lotti I., 2009, Surface and subsurface flow through block ramps, Journal of Irrigation and Drainage Engineering, 135, 3, 366-374

8. Prasad R., 1970, Numerical method of computing flow profiles ASCE, Journal of the Hydraulics Division, 96, HY1, 75-86

9. Soualmia A., Jouini M., Dartus D., 2013, Etude des écoulements dans une mèche en canal, International Journal of PCN, 68, 86-91

10. Stephenson D., 1979, Rockfill in Hydraulic Engineering, Elsevier Science Publishers, New York, USA 